\title{
Tyrosinase Inhibitory Activity and Thermostability of the Flavonoid Complex from Sophora japonica L (Fabaceae)
}

\author{
Jeng-Shiow Lai ${ }^{1,2}$, Chih-Chien Lin ${ }^{1 *}$ and Tsung-Ming Chiang ${ }^{3}$ \\ ${ }^{1}$ Department of Cosmetic Science, Providence University, No. 200, Sec. 7, Taiwan Boulevard, Shalu, Taichung City, 43301, \\ ${ }^{2}$ Department of Beauty Science, Chienkuo Technology University, No. 1 Chiehshou North Road, Changhua City 500, ${ }^{3}$ Panion \& \\ BF Biotech Inc., 16 F, No. 3, Yuan. Chiu St., Nan Gang Dist., Taipei 115, Taiwan, ROC.
}

*For correspondence: Email: chchlin@pu.edu.tw; Tel: +886-4-26328001 ext 15409; Fax: +886-4-26311167.

\begin{abstract}
Purpose: To investigate the tyrosinase inhibitory activity and thermostability of weak acid-treated Sophora japonica L. flavonoid complex (SJ-FC) in different solutions.

Methods: The flavonoid complex of S. japonica was isolated and treated with weak acid to generate SJ-FC. The anti-tyrosinase activities of SJ-FC and well-known tyrosinase inhibitors were compared by mushroom tyrosinase activity assay. The thermostabilities of SJ-FC and other inhibitors in different solution environments for long-term storage were also investigated.

Results: The results indicate that SJ-FC has potent tyrosinase inhibitory activity, and at a concentration of $0.1 \%$, SJ-DC has a tyrosinase inhibitory activity equal to that of $1 \%$ ascorbic acid or hydroquinone. In addition, SJ-FC in both propylene glycol (PG) and glycerol solutions exhibited obvious tyrosinase inhibitory activity. Ascorbic acid and arbutin, two other tyrosinase inhibitors, exhibit $<60 \%$ of their initial activity in both $\mathrm{PG}$ and $\mathrm{H}_{2} \mathrm{O}$ solutions after 6 months of storage. However, SJ-FC stored in $\mathrm{PG}$ and $\mathrm{H}_{2} \mathrm{O}$ solutions retained almost $100 \%$ of its activity over a 6-month period.

Conclusion: SJ-FC is an effective and stable anti-tyrosinase agent and may be used as a function agent in medicines, foods and cosmetics.
\end{abstract}

Keywords: Flavonoid, Sophora japonica L., Tyrosinase inhibitor, Thermostability

Tropical Journal of Pharmaceutical Research is indexed by Science Citation Index (SciSearch), Scopus, International Pharmaceutical Abstract, Chemical Abstracts, Embase, Index Copernicus, EBSCO, African Index Medicus, JournalSeek, Journal Citation Reports/Science Edition, Directory of Open Access Journals (DOAJ), African Journal Online, Bioline International, Open-J-Gate and Pharmacy Abstracts

\section{INTRODUCTION}

The flowers and flower buds of Sophora japonica L. (Fabaceae), widely cultivated in Asia, and also referred to as Styphnolobium japonicum (L.) Schott., are famous traditional Chinese medicines [1]. S. japonica has antioxidant [2,3], anti-inflammatory [4] and anti-platelet activities [5] and can prevent weight gain in highfat diet-induced obese mice [6]. In addition, $S$. japonica contains both anti-hemorrhagic and anti-hemostatic substances. S. japonica reduces the damage caused by cerebral infarction, partly because of its antioxidant and anti-inflammatory activities [1,7].
Flavonoids are secondary metabolites in plants and are of interest to the pharmaceutical, nutritional and cosmetic industries because of their biological activities $[8,9]$. The primary components of $S$. japonica include flavones, tetraglycosides, isoflavones, isoflavone tetraglycosides, triterpene glycosides, phospholipids, alkaloids, amino acids and polysaccharides [10]. However, the major components of the flowers and flower buds of $S$. japonica are rutin, quercetin and rutin-7-Orhamnoside [11]. The structures are presented in Figure 1. Thus, some researchers have proposed that these flavonoid glycosides and 
aglycones are responsible for the most important biological activities of $S$. japonica flowers, including antioxidant and anti-inflammatory activities [11,12].

(A)
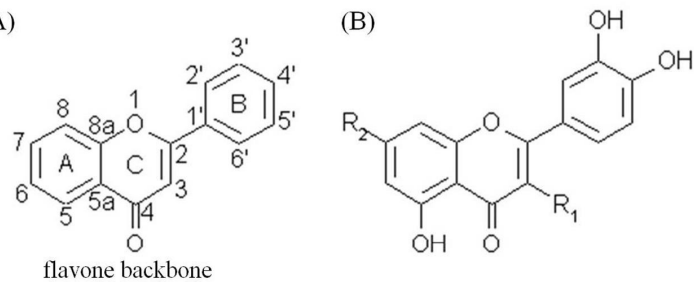

\begin{tabular}{lcc}
\multicolumn{1}{c}{ Compounds } & $\mathrm{R} 1$ & $\mathrm{R} 2$ \\
\hline (1) Rutin & rutinoside & $\mathrm{OH}$ \\
(2) Quercetin & $\mathrm{OH}$ & $\mathrm{OH}$ \\
(3) Rutin-7-O-rhamnoside & rutinoside & rhamnoside \\
\hline
\end{tabular}

Figure 1: Chemical structure of (A) flavone backbone and (B) major flavonoids isolated from Sophora japonica $\mathrm{L}$.

Many plant extracts, some rich in flavonoid aglycones and others rich in flavonoid glycosides, have antioxidant and antiinflammatory activities. Studies have demonstrated that de-glycosylation may increase the absorption of dietary flavones in vivo, enhancing the antioxidant activities of these compounds or modulating inflammatory responses [13,14]. Therefore, adjusting the compositions of flavonoid aglycones and glycosides from natural sources can improve the desired biological functions.

Tyrosinase (EC 1.14.18.1) is the key enzyme catalyses the first two rate-limiting steps of biosynthesis of melanin $[15,16]$. Therefore, tyrosinase is a fine target for inhibition in the search for different types of skin-lightening agents. In practical uses, however, due to the safety and stability concerns, just few tyrosinase inhibitors are used as skin-lightening agents. Thus, the search for new and safe tyrosinase inhibitors is an important issue for the medical and cosmetic industries $[16,17]$.

To our knowledge, there is only one study that showed that a rare compound, $N$-feruloyl- $N$ '-cisferuloyl-putrescine, from $S$. japonica can inhibit a cellular tyrosinase in human epidermal melanocytes [18]. The anti-tyrosinase activity of flavonoid mixtures from the flowers of $S$. japonica has not been reported. Therefore, in this study, we investigated the anti-tyrosinase activity of a weak acid-treated $S$. japonica flavonoid complex (SJ-FC) in different environments. In addition, the thermostabilities of SJ-FC and two other wellknown anti-tyrosinase agents, ascorbic acid and arbutin, were compared.

\section{EXPERIMENTAL}

\section{Materials and chemicals}

Sophora japonica L. [Styphnolobium japonicum (L.) Schott.] was obtained from a local market in Taichung City (Taichung, Taiwan). Glycolic acid, ascorbic acid, hydroquinone, mushroom tyrosinase, L-tyrosine, propylene glycol (PG), glycerol and other chemicals were purchased from Sigma-Aldrich (St. Louis, MO, USA). The $\alpha-$ arbutin and $\beta$-arbutin were purchased from Pentapharm (Basel, Switzerland). Deionized distilled water $\left(\mathrm{ddH}_{2} \mathrm{O}\right)$ for solutions was obtained from a Milli-Q system (Millipore, Bedford, MA, USA).

\section{Preparation of the flavonoid complex}

Fresh flowers of $S$. japonica were washed with pure water and air-dried for 5 days at room temperature. The dehydrated $S$. japonica was homogenized by homogeneizer (Ultra-Turrax T18, IKA, Stauffen, Germany) and extracted with $50 \%$ ethanol at $25{ }^{\circ} \mathrm{C}$ for 30 minutes. The ratio of plant material to liquid was 1: $20(\mathrm{w} / \mathrm{w})$. The supernatant was centrifuged for $15 \mathrm{~min}$ at 3000 rpm and then filtered to remove debris. The filtered flavonoid mixture was then freeze-dried and treated with the weakly-acidic solution ( $0.05 \mathrm{M}$ sulfuric acid in $95 \%$ ethanol) at $40{ }^{\circ} \mathrm{C}$ for $4 \mathrm{~h}$ to generate the $S$. japonica flavonoid complex (SJ-FC) [19]. Sulfuric acid was removed by addition of calcium hydroxide and then the prepared SJ-FC was freeze-dried prior to use.

\section{Tyrosinase activity assay}

To assay the test sample for inhibition of tyrosinase activity, $60 \mu \mathrm{L}$ of test samples in $\mathrm{H}_{2} \mathrm{O}$, propylene glycol or glycerol at different concentrations was mixed with $100 \mu \mathrm{L}$ of $\mathrm{L}$ tyrosine $(1 \mathrm{mM})$ in phosphate-buffered saline $\mathrm{pH}$ 6.8 , propylene glycol or glycerol. Then, $40 \mu \mathrm{L}$ of mushroom tyrosinase solution (100 units/mL) was added to the mixture, which was then incubated for $25 \mathrm{~min}$ at $37^{\circ} \mathrm{C}$. The absorbance was measured spectrophotometrically at $475 \mathrm{~nm}$, and the inhibition of dopachrome formation was calculated as \% inhibition [20]. For the blank group, the complete analytical procedure was followed, including all chemicals and solvents, but no sample was added. The reduction of mushroom tyrosinase activity to a half-maximal inhibitory concentration value is defined as $I_{50}$.

\section{Thermostability analysis}

Solutions of $0.1 \%$ ascorbic acid, arbutin and SJ$\mathrm{FC}$ in $\mathrm{PG}$ or $\mathrm{H}_{2} \mathrm{O}(\mathrm{pH}$ 7) were placed in glass 
bottles and kept in the dark. These samples were maintained at room temperature $\left(25^{\circ} \mathrm{C}\right)$ in an incubator. At predetermined intervals (from 0 to 180 days), the solutions were analyzed using the previously described tyrosinase inhibition assay to determine the stability of the inhibitory activities of these compounds.

\section{Statistical analysis}

The quantitative data for the present study were analyzed using Student's $t$-tests and are presented as mean \pm SEM for three independent assays.

\section{RESULTS}

\section{Preparation of SJ-FC}

The yield of flavonoids from $S$. japonica flowers was about $0.502 \%$ (w/w, 5.02g SJ-FC from $1000 \mathrm{~g}$ dried flowers of S. japonica).

\section{Tyrosinase inhibitory activity of various tyrosinase inhibitors and SJ-FC}

First, we compared the anti-tyrosinase activity of SJ-FC $(0.1 \%)$ with those of several tyrosinase inhibitors ( $1 \%)$, including glycolic acid, $\beta$-arbutin, $\alpha$-arbutin, ascorbic acid and hydroquinone in $\mathrm{H}_{2} \mathrm{O}$ solution. The results are shown in Figure 2. It is clear that all anti-tyrosinase agents have inhibitory activities against mushroom tyrosinase. In addition, except for glycolic acid, all tested inhibitors at a $1 \%$ concentration have inhibitory activities greater than $70 \%$ relative to the control (blank, no sample was added). The inhibition of glycolic acid was approximately $37 \%$. Ascorbic acid and hydroquinone at a concentration of $1 \%$ and SJ-FC at a concentration of $0.1 \%$ had the greatest tyrosinase inhibitory activity (Figure 2).

The calculated half-maximal inhibitory concentration $\left(\mathrm{IC}_{50}\right)$ value of SJ-FC for tyrosinase activity in $\mathrm{H}_{2} \mathrm{O}$ solution is $3.12 \mathrm{mg} / \mathrm{mL}$ (Table 1). Therefore, we can suppose that SJ-FC is a potent tyrosinase inhibitory agent.

\section{Tyrosinase inhibitory activity of SJ-FC in PG and glycerol solutions}

The results in Figure 3 show that SJ-FC at concentrations of 0.01 to $0.1 \%$ inhibits tyrosinase in both polyol environments. Moreover, $0.1 \%$ SJ-FC suppressed tyrosinase almost completely in PG (Figure 3A) and glycerol (Figure $3 \mathrm{~B}$ ) solutions. Although the inhibition of SJ-FC at higher concentrations in both solutions did not differ significant, the results indicated that SJ-FC in PG has greater tyrosinase inhibitory activity (higher than $60 \%$ inhibition) at lower concentrations $(0.01 \%$ SJ-FC, Figure $3 \mathrm{~A}$ and $3 B)$. $P G$ is a thus better polyol solution for the anti-tyrosinase function of SJ-FC.

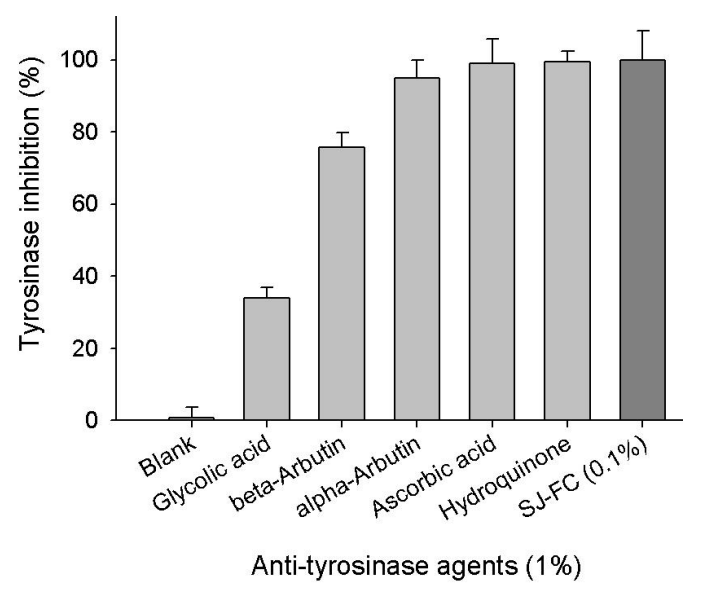

Figure 2: Tyrosinase inhibitory activities of various tyrosinase inhibitors $(1 \%)$ and SJ-FC $(0.1 \%)$ in $\mathrm{H}_{2} \mathrm{O}$ solution.
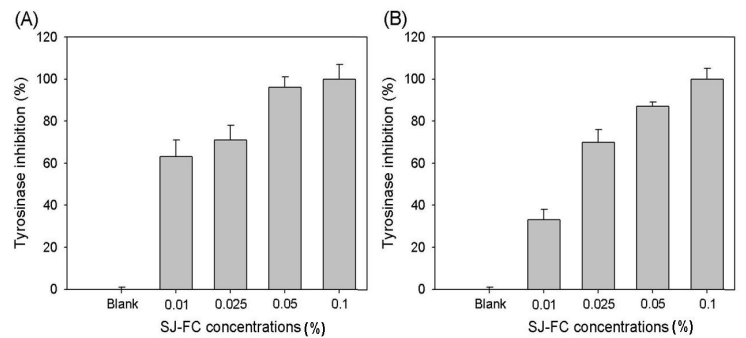

Figure 3: (A) Tyrosinase inhibitory activity of SJ-FC in PG solutions; (B) tyrosinase inhibitory activity of SJ-FC in glycerol solutions.

\section{Stability of ascorbic acid, arbutin and SJ-FC in PG and aqueous solutions}

The results of this assay are presented in Figures $4 \mathrm{~A}, \mathrm{~B}$ and $\mathrm{C}$. The results indicate that ascorbic acid decomposes in both $\mathrm{PG}$ and $\mathrm{H}_{2} \mathrm{O}$ solutions. When the storage time is greater than 6 months, the inhibitory activity of ascorbic acid was decreased to $<60 \%$ of its initial value (Figure 4A).

A similar result is presented in Figure $4 \mathrm{~B}$ for arbutin. Arbutin's inhibitory function in $P G$ and $\mathrm{H}_{2} \mathrm{O}$ solutions decreased to $39 \%$ and $21 \%$, respectively, of its initial activity after 6 months of storage. In addition, the stability of arbutin in PG is better than that in $\mathrm{H}_{2} \mathrm{O}$ solution (Figure $4 \mathrm{~B}$ ).

Figure $4 \mathrm{C}$ shows the stability of SJ-FC in $P G$ and $\mathrm{H}_{2} \mathrm{O}$ solutions. Over the 6-month period storage, SJ-FC in $P G$ and $\mathrm{H}_{2} \mathrm{O}$ solutions retained almost all of its original activity, higher than $97 \%$ inhibition. This result indicates that SJ-FC is a stable tyrosinase inhibitor in both $P G$ and $\mathrm{H}_{2} \mathrm{O}$ solutions. 
(A)

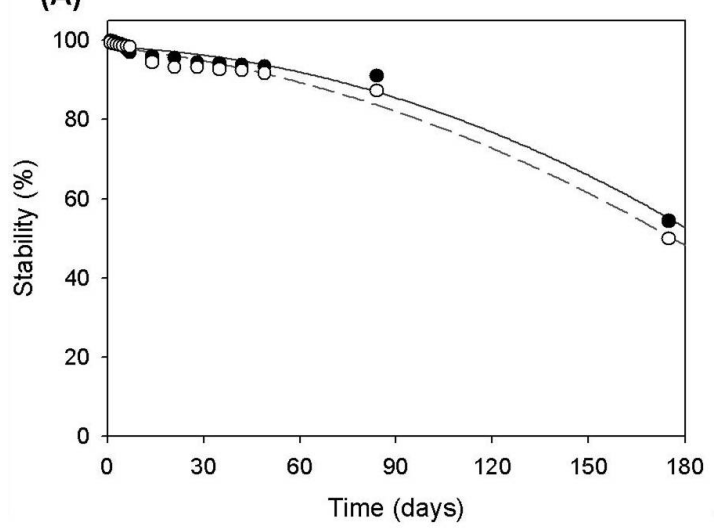

(B)

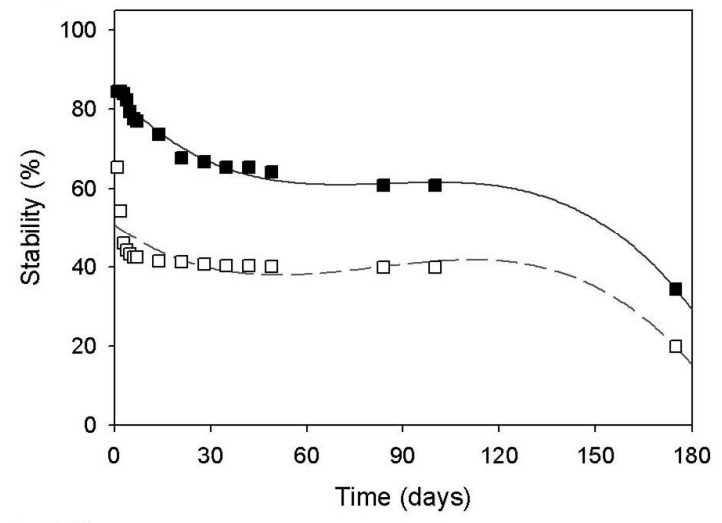

(C)

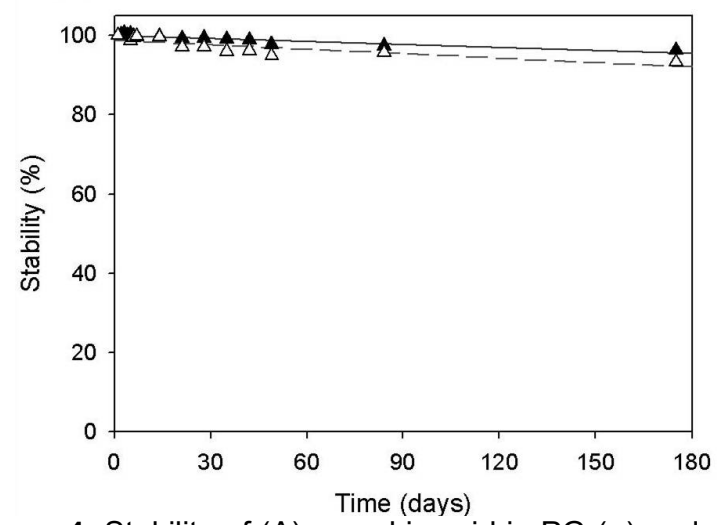

Figure 4: Stability of (A) ascorbic acid in $P G(\bullet)$ and $\mathrm{H}_{2} \mathrm{O}(\circ)$ solutions; (B) arbutin in PG (ם) and $\mathrm{H}_{2} \mathrm{O}(\square)$ solutions; (C) SJ-FC in PG ( $(\Delta)$ and $\mathrm{H}_{2} \mathrm{O}(\Delta)$ solutions.

\section{DISCUSSION}

The main components of SJ-FC are rutin, quercetin and rutin-7-O-rhamnoside. We used the weakly-acidic solution treatment of its flavonoid mixture to obtain SJ-FC that has several functional constituents, including flavone glycosides and the aglycones and glycosides formed by hydrolysis of the flavone glycosides. Moreover, we suppose that weakly-acidic solution treatment also provides a procedure with broken of some unstable bonds to produce a relative stable flavonoid complex [19].
Additionally, the anti-tyrosinase activity of several important flavonoids, including rutin and quercetin, had been established by some previous studies [21-23]. For this reason, we can assume that the anti-tyrosinase activity of SJ-FC is given by these important components. Our results also demonstrate that SJ-FC is an effective and stable tyrosinase inhibitor.

This result also concludes that SJ-FC has potent anti-tyrosinase activity. The activity of SJ-FC is better than that of other common used tyrosinase inhibitors. Moreover, $\alpha$-arbutin exhibits higher anti-tyrosinase activity than $\beta$-arbutin (Figure 2). This observation is similar to that of an earlier study showing that $\alpha$-arbutin has greater tyrosinase inhibitory activity than $\beta$-arbutin [24].

When used as an anti-tyrosinase agent in medicines, foods and cosmetics, this inhibitor might exert its activity in aqueous environments, such as aqueous solutions, polyol-containing solutions and various types of emulsions. In addition, formulations used in medicines, foods and cosmetics frequently include $P G$ and glycerol. Therefore, if SJ-FC is a functional inhibitor when dissolved in PG and glycerol, it can be used in more applications in the future. The results also indicate that SJ-FC in both PG and glycerol solutions has good tyrosinase inhibitory activity (Figure 3).

The stability of active ingredients for medicines, foods and cosmetics is an important factor in their use. Some potent anti-tyrosinase agents, such as kojic acid, arbutin and deoxyarbutin, are unstable in aqueous environments [25-27]. Thus, we compared the stability of SJ-FC with the stabilities of two well-known and frequently used tyrosinase inhibitors, ascorbic acid and arbutin, using a tyrosinase inhibitory activity-based method in $P G$ and $\mathrm{H}_{2} \mathrm{O}$ solutions. Our results indicate that SJ-FC is a potent and stable antityrosinase agent. This result suggests that SJ-FC can act as a stable tyrosinase inhibitor in many types of environments (formulations) that involve long-term storage. Hence, this feature of SJ-FC will facilitate its use as ingredient in many applications. Moreover, based on these results, we can state that ascorbic acid and arbutin are very unstable in both $P G$ and $\mathrm{H}_{2} \mathrm{O}$ environments (Figures $4 A$ and $B$ ).

\section{CONCLUSION}

SJ-FC is a potent tyrosinase inhibitory agent which, at a concentration of $0.1 \%$, has greater tyrosinase inhibitory activity than $1 \%$ ascorbic acid or hydroquinone. In addition, SJ-FC in both PG and glycerol solutions has potent tyrosinase inhibitory activity. However, at lower 
concentrations $(0.01 \% \mathrm{SJ}-\mathrm{FC}), \mathrm{PG}$ is a better polyol solution for the anti-tyrosinase function of SJ-FC. Ascorbic acid and arbutin exhibit $<60 \%$ of their initial activity after storage in either PG and $\mathrm{H}_{2} \mathrm{O}$ solutions for 6 months. However, SJ-FC stored in $\mathrm{PG}$ and $\mathrm{H}_{2} \mathrm{O}$ solutions retains almost $100 \%$ of its activity over the 6 -month period. Therefore, SJ-FC is a potent and stable antityrosinase agent and may have various applications for medicines, foods and cosmetics in the future.

\section{ACKNOWLEDGEMENT}

The authors are grateful for the financial support by Panion \& BF Biotech Inc provided to Professor Lai and the financial support from the National Science Council of the Republic of China (ROC) awarded to C.-C. Lin (NSC992313-B-126-002- MY3).

\section{REFERENCES}

1. Chen HN, Hsieh CL. Effects of Sophora japonica flowers (Huaihua) on cerebral infarction. Chin Med 2010; 5: 34: 1-4.

2. Paniwnyk L, Beaufoy E, Lorimer JP, Mason TJ. The extraction of rutin from flower buds of Sophora japonica. Ultrason Sonochem 2001; 8(3): 299-301.

3. Tang YP, Li YF, Hu J, Lou FC. Isolation and identification of antioxidants from Sophora japonica. J Asian Nat Prod Res 2002; 4(2): 123128.

4. Zhang L, Ravipati AS, Koyyalamudi SR, Jeong SC, Reddy N, Smith PT, Bartlett J, Shanmugam K, Munch G, Wu MJ. Antioxidant and antiinflammatory activities of selected medicinal plants containing phenolic and flavonoid compounds. $J$ Agric Food Chem 2011; 59(23): 12361-12367.

5. Kim JM, Yun-Choi HS. Anti-platelet effects of flavonoids and flavonoid-glycosides from Sophora japonica. Arch Pharm Res 2008; 31(7): 886-890.

6. Park KW, Lee JE, Park KM. Diets containing Sophora japonica $L$. prevent weight gain in high-fat dietinduced obese mice. Nutr Res 2009; 29(11): 819824.

7. Lao CJ, Lin JG, Kuo JS, Chao PD, Cheng CY, Tang NY, Hsieh CL. Microglia, apoptosis and interleukin1 beta expression in the effect of Sophora japonica $L$. on cerebral infarct induced by ischemiareperfusion in rats. Am J Chin Med 2005; 33(3): 425-438.

8. Aparicio S. A systematic computational study on flavonoids. Int J Mol Sci 2010; 11(5): 2017-2038.

9. Kostić DA, Velicković JM, Mitić SS, Mitić MN, Randelović SS. Phenolic content, and antioxidant and antimicrobial activities of Crataegus Oxyacantha $L$ (Rosaceae) fuit extract from southeast Serbia. Trop J Pharm Res 2012; 11(1): 117-124.

10. Wang JH, Lou FC, Wang YL, Tang YP. A flavonol tetraglycoside from Sophora japonica seeds. Phytochemistry 2003; 63(4): 463-465.

11. Kite GC, Veitch NC, Boalch ME, Lewis GP, Leon CJ, Simmonds MS. Flavonol tetraglycosides from fruits of Styphnolobium japonicum (Leguminosae) and the authentication of Fructus Sophorae and Flos Sophorae. Phytochemistry 2009; 70(6): 785-794.

12. Chu $Q, F u L, W u T, Y$ J J. Simultaneous determination of phytoestrogens in different medicinal parts of Sophora japonica L. by capillary electrophoresis with electrochemical detection. Biomed Chromatogr 2005; 19(2): 149-154.

13. Hostetler $G$, Riedl $K$, Cardenas $H$, Diosa-Toro $M$, Arango D, Schwartz S, Doseff Al. Flavone deglycosylation increases their anti-inflammatory activity and absorption. Mol Nutr Food Res 2012; 56(4): 558-569.

14. Murota K, Matsuda N, Kashino Y, Fujikura $Y$, Nakamura $T$, Kato $Y$, Shimizu R, Okuyama S, Tanaka $H$, Koda $T$ et al. alpha-Oligoglucosylation of a sugar moiety enhances the bioavailability of quercetin glucosides in humans. Arch Biochem Biophys 2010; 501(1): 91-97.

15. Chang TS. Natural Melanogenesis Inhibitors Acting Through the Down-Regulation of Tyrosinase Activity. Materials 2012; 5(9): 1661-1685.

16. Gillbro JM, Olsson MJ. The melanogenesis and mechanisms of skin-lightening agents--existing and new approaches. Int J Cosmet Sci 2011; 33(3): 210-221.

17. Lin CC, Yang $\mathrm{CH}$, Chang NF, Wu PS, Chen YS, Lee SM, Chen CW. Study on the stability of deoxyArbutin in an anhydrous emulsion system. Int $J$ Mol Sci 2011; 12(9): 5946-5954.

18. Lo $Y H$, Lin $R D$, Lin $Y P$, Liu $Y L$, Lee $M H$. Active constituents from Sophora japonica exhibiting cellular tyrosinase inhibition in human epidermal melanocytes. J Ethnopharmacol 2009; 124(3): 625629.

19. Kim DO, Padilla-Zakour OI, Griffiths PD. Flavonoids and Antioxidant Capacity of Various Cabbage Genotypes at Juvenile Stage. J Food Sci 2004; 69(9): C685-C689.

20. Lin CC, Yang $\mathrm{CH}$, Wu PS, Kwan CC, Chen YS. Antimicrobial, anti-tyrosinase and antioxidant activities of aqueous aromatic extracts from fortyeight selected herbs. J Med Plants Res 2011; 5(26): 6203-6209.

21. Chen $Q X, K u b o$ I. Kinetics of mushroom tyrosinase inhibition by quercetin. J Agric Food Chem 2002; 50(14): 4108-4112.

22. Si YX, Yin SJ, Oh S, Wang ZJ, Ye S, Yan L, Yang JM, Park YD, Lee J, Qian GY. An integrated study of tyrosinase inhibition by rutin: progress using a computational simulation. J Biomol Struct Dyn 2012; 29(5): 999-1012.

23. Xie $L P$, Chen $Q X$, Huang $H$, Wang $H Z$, Zhang $R Q$. Inhibitory effects of some flavonoids on the activity of mushroom tyrosinase. Biochemistry (Mosc) 2003; 68(4): 487-491.

24. Funayama $M$, Arakawa $H$, Yamamoto $R$, Nishino $T$, Shin $T$, Murao S. Effects of alpha- and beta-arbutin on activity of tyrosinases from mushroom and mouse melanoma. Biosci Biotechnol Biochem 1995; 59(1): 143-144.

25. Noh JM, Kwak SY, Seo HS, Seo JH, Kim BG, Lee YS. Kojic acid-amino acid conjugates as tyrosinase inhibitors. Bioorg Med Chem Lett 2009; 19(19): 5586-5589.

26 Sugimoto K, Nomura K, Nishimura T, Kiso T, Sugimoto $K$, Kuriki T. Syntheses of alpha-arbutin-alphaglycosides and their inhibitory effects on human tyrosinase. J Biosci Bioeng 2005; 99(3): 272-276.

27. Yang $\mathrm{CH}$, Chen YS, Lai JS, Hong WW, Lin CC. Determination of the thermodegradation of deoxyArbutin in aqueous solution by high performance liquid chromatography. Int $\mathrm{J} \mathrm{Mol} \mathrm{Sci}$ 2010; 11(10): 3977-3987. 